Biomath Communications
Biomath Forum
www.biomathforum.org/biomath/index.php/conference

\title{
Biomathematical Conferences and Schools for Young Scientists at the Bulgarian Academy of Sciences]
}

\author{
Roumen Anguelov, Svetoslav Markov
}

\begin{abstract}
This is a brief report on some international meetings in the field of biomathematics and scientific computations organized within the frames of the Bulgarian Academy of Sciences. Most meetings involved both a scientific conference and a School for Young Scientists aiming to attract doctoral students and young scientists and to promote the integration between learning and research activities.
\end{abstract}

\section{Introduction}

Biomathematics is an established field of education and research, also known as mathematical biology or theoretical biology [23, [44. Biomathematics research involves applications of mathematics in life sciences as well as mathematics research applied to or motivated by biological studies. Biomathematicians develop and apply mathematical and computational tools to the study of phenomena in the broad fields of biology, ecology, medicine, biotechnology, bioengineering, environmental science, etc.

Related fields. The interaction between biology and mathematics goes in two opposite directions: i) novel contemporary mathematical tools and

${ }^{1}$ Citation: R. Anguelov, S. Markov, Biomathematical Conferences and Schools for Young Scientists at the Bulgarian Academy of Sciences. Biomath Communications 1/2 (2014) http://dx.doi.org/10.11145/j.bmc.2014.02.261 
models are increasingly introduced in life sciences, and, ii) biological problems require and generate new mathematical results and stimulate the development of novel mathematical theories and computational tools, such as computer algebra systems, enclosure methods and interval analysis etc. [20], 23].

Uncertainty in bioprocesses. A main characteristic of biological systems is uncertainty, due to the use of simplified models, variation with time of parameters, unpredictable phenomena, inexact measurements, intrinsic sensitivity of the system, etc. Characterization of uncertainty and its consideration in subsequent analysis is important for understanding bioprocesses, the identification of state variables and the design of effective control strategies. Researchers in life sciences often underestimate uncertainty. Although formal methods for dealing with uncertainty have been developed over the last decades, little effort has been invested so far to produce results concerning guaranteed characterization of uncertainty, guaranteed modelling and simulation, guaranteed optimization, experiment design, robust control, etc. [21].

For model identification of biological processes involving short uncertain records and unstable solutions (as is often the case whenever enzyme reactions are present) it seems that novel mathematical tools, such as differential inclusions, set-valued analysis, viability analysis, enclosure methods, interval analysis, stochastic methods, will play a major roles in the future. Such methods may require some additional effort from experimental scientists in providing bounds for the measured quantities.

The management of uncertainty in biological systems requires an interaction and transfer of knowledge between different fields involved, such as biological modelling, bioprocess applications, CAS, enclosure methods, interval analysis, validated numerical analysis, robust control. The integration of experimental and computational research is helpful for the understanding of complex biological systems.

Education in Biomathematics. University programs in areas defined as biomathematics, theoretical biology, bioinformatics, computational biology, exist worldwide. Transformation of graduate, undergraduate and postgraduate educational programs has been recently actively discussed in many countries [39]-41]. It is widely recognized that learning biomathematics is now an important part of human education. 
In this report we summarize information on a series of international academic conferences in the field of biomathematics and related fields held in Bulgaria within the Bulgarian Academy of Sciences. Most of the meetings were accompanied by Schools for Young Scientists. The meetings were focused in the area of mathematical modelling in biology and related fields such as ecology, biotechnology, physiology, medicine etc. From the point of view of mathematical and proramming tools the organizers welcomed advance mathematical and computational tools such as verification methods, interval analysis, computer algebra systems etc. The meetings sometimes were focused more on biomathematical applications and were named "Biomathematics (Biomath)" and sometimes were focused on mathematical tools and were named "Mathematical Modelling and Scientific Computation (MMSC)". Altogether over ten international meetings were organized in the period 1995-2015. Since 2011 four annual international academic meetings in the field of biomathematics were held in Bulgaria under the name "Biomath" [26].

Remark. The enlisted below meetings do not cover the whole spectrum of scientific meetings related to biomathematics organized within the Bulgarian Academy of Sciences. For example, the meetings organized by the Probability and Statistics Department at IMI-BAS, the biomathematical meetings organized by the Institute of Mechanics and by the Institute of Biophysics and Biomedical Engineering at BAS are not mentioned.

\section{Conferences}

In this section we enlist chronologically a number of meetings related to biomathematical sciences organized within the Bulgarian Academy of Sciences. In the list we include several mathematical conferences that involved biomathematical workshops and special sessions.

SCAN-1990. The Second International Symposium on Computer Arithmetic, Scientific Computaion and Mathematical Modelling (SCAN), took part September 23-28, 1990, in Albena, Bulgaria. Nearly two hundred scientists from 14 countries participated in the meeting; 33 selected and peer-reviewed papers were published [2].

MMSC-1993. The International Conference on Mathematical Modelling and Scientific Computation was held in Sozopol, September 14-18, 
1993. Two book collections were published [3], 4]. An international workshop on "Mathematical Modelling and Information Systems in Biology, Ecology and Medicine" was organized within the conference. Part II of [4] entitled "Mathematical Models in Biology and Medicine" contains 26 papers papers and extended abstracts related to lectures presented at this workshop.

BIOMATH-1995. The International Symposium and Young Scientists School on Mathematical Modelling and Information Systems in Biology, Ecology and Medicine (BIOMATH-1995) was organized by the Research Group for Mathematical Modelling in Biology at the Bulgarian Academy of Sciences and was held in Sofia, Bulgaria from August 23 to 27, 1995 [5]. Programm Committee: P. Antonelli (Canada), P. Auger (France), Y. Cherruault (France), J.-L. Gouze (France), J. Li (USA), V. Krivan (Czech Republic), P. Maini (UK), J. Milota (Czech Republic), W. Miranker (USA), J. Norton (UK), R. Tsanev (Bulgaria), Z. Zlatev (Denmark). BIOMATH-1995 was attended by 50 scientists from 16 different countries; 35 scientific lectures were presented and 25 papers were selected for publication [6], [7, 8].

Destobio-1997. The international conference on "Deterministic and Stochastic Aspects of Biointeraction (DESTOBIO'97)" took place in Sofia, Bulgaria, from August 28 to 31, 1997. About 140 researchers from many fields and nationalities participated at this meeting [22]. There were 60 submitted papers, 34 were published in two consecutive Special issues of the Elsevier journal "Mathematical Biosciences" [9], [10]. Programm Committee: J. Cushing (USA), O. Diekmann (The Netherlands), M. Gyllenberg (Finland), M. Iannelli (Italy), P. Jagers (Sweden), M. Kimmel (USA), T. Kostova-Vassilevska (Chair, Bulgaria), J. Li (USA), F. Milner (USA), H. Smith (USA), A. Wakolbinger (Germany), N. Yanev (Bulgaria). Invited speakers: J. Cushing, O. Diekmann, M. Iannelli, P. Jagers, M. Kimmel, J. Li, F. Milner.

NMA-1998 Minisymposium "Solving Linear Systems with Error Control" at the 4th International Conference on Numerical Methods and Applications (NMA'98), August 23, 1998, Sofia. For a report on the minisymposium see Reliable Computing 5 (1), 97-100, 1999 [11].

RC\&IA-1999. The International Workshop on Reliable Computing and Interval Algebra (RC\&IA) took place in Sozopol, 27-29.09.1999; par- 
ticipants from Bulgaria, France, Germany, Poland, Russia, Turkey [12].

MMSC-2001. Minisymposium on "Mathematical Modelling and Scientific Computations (MMSC-2001)" was organised within the 30-th Spring Conference of the Union of Mathematicians in Bulgaria, 8-11 april, 2001, Borovets. The presentations of this minisimposium are included in the proceedings of the conference [13].

MMSC-2002. Minisymposium on "Mathematical Modelling and Scientific Computations (MMSC-2002)" was organised within the 31-st Spring Conference of the Union of Mathematicians in Bulgaria, 2-6 april, 2002, Borovets. The presentations of this minisimposium are included in the proceedings of the conference [14].

NM\&A-2002 Minisymposium on "Control and Uncertain Systems" at the Fifth International Conference on Numerical Methods and Applications, 20-24.08.2002. Seven peer reviewed papers published in LNSC 2542 Springer 2003 [15].

Scicom-2003 Minisymposium on "Set-valued Numerics and Reliable Computing" at 4th International Conference on "Large-Scale Scientific Computations" 4-8.06.2003, Sozopol [19]

ACA-2006 Special Session "Interaction Between Computer Algebra and Interval Computations" at the 12th International Conference on Applications of Computer Algebra (ACA 2006), June 26-29, 2006, Varna, Bulgaria [16]. Such special sessions are orgnized also at the ACA-2008, ACA-2009, ACA-2010 and ACA-2012 meetings.

NM\&A-2006 Minisymposium on "Uncertain/Control Systems and Reliable Numerics" at the 6th International Conference on Numerical Methods and Applications, Borovets, Bulgaria, August 20-24, 2006. About twenty participants (six from abroad) submitted are twoelf (12) peer reviewed papers published in LNSC 4310 Springer 2007 [17].

MMSC-2009. Minisymposium on "Mathematical Modelling and Scientific Computations (MMSC-2009)" was organized in Velingrad 2009 with about 30 participants. A Special issue of Serdica Journal of Computing containing twelve articles were publiched [18]

Biomath-2011 [28]. The international conference on Mathematical Methods and Models in Biosciences took place from 15 June to 18 June 
2011 at the campus of the Bulgarian Academy of Sciences in Sofia, Bulgaria. The event attracted about hundred interdisciplinary participants from a dozen countries. Keynote speakers: A. S. Ackleh (USA), R. Alt (France), V. Beschkov (Bulgaria), Y. Dumont (France), R. V. Gueorguieva (USA), H. Kojouharov (USA), W. A. Lodwick (USA), J. Lubuma (RSA), S. Margenov (Bulgaria), N. Yanev (Bulgaria). The Biomath-2011 meeting resulted in the publication of two special issues of the journals "Computers and Mathematics with Applications" 29] and "Biotechnology \& Biotechnological Equipment" 32.

Biomath-2012 [30]. The international conference on Mathematical Methods and Models in Biosciences (Biomath-2012) took place from 17 June to 22 June 2012 at the campus of the Bulgarian Academy of Sciences in Sofia, Bulgaria. Keynote speakers: N. Dimitrova (Bulgaria), J. Z. Farkas (UK), P. Hinow (USA), K. Ivanov (Bulgaria), C. Timofte (Romania), S. Schnell (USA), P. Hingley (Germany). The Biomath-2012 meeting resulted in the publication of a special issue of the journal "Computers and Mathematics with Applications" [31].

Biomath-2013 33]. The annual international conference on Mathematical Methods and Models in Biosciences (Biomath-2013) took place from 16 June to 21 June 2013 at the campus of the Bulgarian Academy of Sciences in Sofia, Bulgaria. Keynote speakers: B. Asquith (UK), S. D. Bolboaca (Romania), C. Chavez-Castillo (USA), N. Cogan (USA), G. Rost (Hungary), V. Rom-Kedar (Israel), A. Niemi (Sweden), M. R. Roussel (Canada), M. Shillor (USA), S. Sonner (Spain). The Biomath-2013 meeting resulted in the publication of a special issue of the journal "Computers and Mathematics with Applications".

Biomath-2014 35]. The annual international conference on Mathematical Methods and Models in Biosciences (Biomath) took place from 22 June to 27 June 2014 at the campus of the Bulgarian Academy of Sciences in Sofia, Bulgaria. Over 90 participants from 22 countries attended the meeting. More than 30 of them were young scholars with presentations15 from Bulgaria, seven from USA. six from South Africa. Plenary lecture: A. Karshikoff (Bulgaria), keynote lectures: N. Chitnis (Switzerland), P. Erdi (Hungary), V. Klika (Czech Republic), M. Martcheva (USA), J. J H Miller, Ireland; S. G. Nikolov (Bulgaria), A. Pashov (Bulgaria), U. Ufuktepe (Turkey). The Biomath-2014 meeting resulted in the submission of 
more than twenty papers for publication in a special issue of the Elsevier journal "Mathematics and Computation with Simulations (MATCOM)" (papers are now in review).

Biomath-2015 [36]. The annual international conference on Mathematical Methods and Models in Biosciences (Biomath) will take place from 14th to 19th June 2015 at the University centre Bachinovo, Blagoevgrad, Bulgaria, and will be hosted by the South-West University "N. Rilski". A satellite conference will take place in South Africa three weeks later.

The conference series Biomath is devoted to recent research in biosciences based on applications of mathematics as well as mathematics applied to or motivated by biological applications. It is a multidisciplinary meeting forum for researchers who develop and apply mathematical and computational tools to the study of phenomena in the broad fields of biology, ecology, medicine, biotechnology, bioengineering, environmental science, etc.

\section{Schools for Young Scientists}

Almost all enlisted above conferences involved a School for Young Scientists. During the years the organization of the meetings gradually evolved and reached an original format allowing a tight intergration between science and education.

MMSC-1990 Summer School. The Summer School on Mathematical Modelling and Scientific Computation (MMSC-90) was held 23-28 September, 1990, in Albena, Bulgaria. parallel to the SCAN-1990 International Symposium. More than 50 participants from eight countries took part in the school; nine tutorial lectures, 18 talks and 28 posters were presentated; 28 selected papers were published in a book collection [1]

MMSC-1993 Summer School. The Summer School on Mathematical Modelling and Scientific Computation (MMSC-93) was held 14-17 September in Sozopol parallel to the MMSC-93 International Conference. 42 selected papers were published in a book collection [4].

Biomath-2011-12. The 2011 and the 2012 Biomath conferences involved an one-day School for Young Scientists (SYS) organized mainly in 
a traditional form. Established lecturers were invited to present tutorial lectures for the participating $(\mathrm{PhD})$ students.

Biomath-2013-14. At the 2013 and 2014 Biomath conferences we experimented a novel original integration format of the SYS with the scientific conference. No particular tutorial lectures were offered at these meetings. Instead, the plenary and keynote lecturers were asked to present their lectures in a suitable form for the broad audience and the SYS participants were asked to attend these lectures and were encouraged to ask questions. Also, the presentations by the SYS participants were assessed both by their peers and a jury amongst the keynote lecturers and some of the established scholars and were awarded respectively. Accordingly the members of the jury were asked to listen to students presentations. In addition several of the established scholars known to have PhD students were asked to present "hot topics \& open problems" and act as moderators of the discussions during this presentation. In this way the School for young scientists was fully integrated with the conference in the sense that both young scientists and many of the established scholars participated in the learning process. At an earliest occasion the participating young scientists were gathered at a meeting and were instructed about the specific arrangement of the conference.

\section{Publications}

Publications are an important final outcome and goal of the conference meetings. Most of the enlisted meetings produced book or journal collections of scientific papers. The Biomath conferences resulted in a significant number of original research articles [27. About half of the conference papers are published in special issues of the Elsevier journals "Mathematical Biosciences" and "Computers and Mathematics with Applications". Other journals publishing Biomath special issues are "J.UCS", "B\&BE", "MATCOM", "BIOMATH" and "Biomath Communications", all of them using a peer-to-peer review process. Journal "BIOMATH" published by Biomath Forum [26] follows the highest standards of contemporary academic publishing of original scholar articles. Journal "Biomath Communications" [26] also publishes original articles and in addition publishes theses, course projects, review articles, open problems and recent advances, educational materials, abstracts of lectures and posters, news and announcements re- 
lated to the field of Biomathematics, etc. "Biomath Communications" aims at supporting the idea of integrating learning and research acting as a forum for young researchers to communicate their scientific projects, dissertations and related problems.

\section{Conclusion}

In this work we share our experience with the organization of the scientific conference series Biomath and the accompanying educational and publication activities. We have effectively integrated these three activities to optimize the final outcome, namely possibly quick publishing of good quality presentations.

We hope that the presented report may be useful for colleagues who are planning to attend future Biomath meetings. The report may also be useful for colleagues who are ready and willing to invest time and efforts for organizing similar academic events. Surely these colleagues must have some experience in conference organization and follow certain basic rules, cf. [24, [?].

Acknowledgements. The Biomath-2014 was financially supported by the Buylgarian Ministry of Education and Science, by the American NSF and by the NRF of the Republic of South Africa.

\section{References}

[1] Andreev, A., I. Dimov, S. Markov, Ch. Ullrich (Eds.), Mathematical Modelling and Scientific Computations, Publ. House of the Bulg. Acad. of Sci., Sofia, 1991.

[2] Kaucher, E., S. Markov, G. Meyer (Eds.), Computer Arithmetic, Scientific Computation and Mathematical Modelling, IMACS Annals on computing and applied mathematics 12, Baltzer Sci. Publ., Basel, 1991.

[3] Markov, S. (Ed.), Scientific Computation and Mathematical Modelling, Datecs Publ., Sofia, 1993. 
[4] Popova, E. D. (Ed.), Mathematical Modelling: Methodology, Software Tools and Applications, Datecs Publ., Sofia, 1993.

[5] Biomath 1995 Conference website. http://www . biomath.bg/1995/BIOMATH95.php

[6] BIOMATH-95 (Guest editors: Ch. Ullrich and S. Markov), Computers and Mathematics with Applications 32 (11), Dec 1996, Proc. Int. Symposium and Young Scientists School on Mathematical Modelling and Information Systems in Biology, Ecology and Medicine BIOMATH-95.

http://www.sciencedirect.com/science/

journal/08981221/32/11

[7] Markov, S., Ch. Ullrich (Guest Editors), BIOMATH-95, Journal of Universal Computer Science (J. UCS) 2, 2, February 1996, 58-95. http://dx.doi.org/10.3217/jucs-002-02

[8] Candev, M., (Ed.) Lecture Notes on Biomathematics and Bioinformatics'95, DATECS Publ., pp. 114, ISBN 954-613-006-0.

[9] Mathematical Biosciences 156 (1-2), 1-342 (1 March 1999), special issue: "Epidemiology, Cellular Automata and Evolution".

[10] Mathematical Biosciences, 157 (1-2), 1-376 (15 March 1999), special issue: "Deterministic Models with Applications in Population Dynamics and Other Fields of Biology".

[11] Dimitrova, N., S. Markov, Ch. Ullrich, Solving Linear Systems with Error Control: Minisymposium within the 4th International Conference NMA'98, Reliable Computing 5 (1), 97-100, 1999.

[12] International Workshop on Reliable Computing and Interval Algebra (RC\&IA-99) Sozopol, 27-29.09.1999 http://www.math.bas.bg/ bio/RC\&IA'99/

[13] Minisymposium on Mathematical Modelling and Scientific Computations (MMSC-2001). In: Mathematical Modelling and Mathematical Education 2001, Proc. 30-th Spring Conf. Union of Mathematicians in Bulgaria, 8-11 april, 2001, Borovets, 432-467 
http://www.math.bas.bg/smb/2001_2003_PK

/2001/tom_2001.html

[14] Minisymposium on Mathematical Modelling and Scientific Computations (MMSC-2002). In: P. Popivanov (ed.) Mathematical Modelling and Mathematical Education 2002, Proc. 31-st Spring Conf. Union of Mathematicians in Bulgaria, 3-6 april, 2002, Borovets, 320-391. http://www.math.bas.bg/smb/2001_2003_PK/ 2002/tom_2002.html

[15] NM\&A-2002 Minisimposium "Control and Uncertain Systems"; Dimov, I., I. Lirkov, S. Margenov, Z. Zlatev (Eds.): Numerical Methods and Applications, 5th International Conference, NMA 2002, Borovets, Bulgaria, August 20-24, 2002, Lecture Notes in Computer Science 2542 Springer 2003, ISBN 3-540-00608-7

http://www.sigmod.org/publications/ $\mathrm{dblp} / \mathrm{db} / \mathrm{conf} / \mathrm{nma} / \mathrm{nma2002}$.html

[16] ACA-2006 Special Session at 12th International Conference on Applications of Computer Algebra (ACA 2006) June 2629, 2006, Varna, Bulgaria http://www. math.bas.bg/ẽpopova/ACA2008/ http://www. math.bas.bg/ẽpopova/ACA2009/ http://www.math.bas.bg/ẽpopova/ACA2010/ http://www.math.bas.bg/ẽpopova/ACA2012/

[17] NM\&A-2006 Minisimposium "Uncertain/Control Systems and Reliable Numerics"; Todor Boyanov, Stefka Dimova, Krassimir Georgiev, Geno Nikolov (Eds.): Numerical Methods and Applications, 6th International Conference, NMA 2006, Borovets, Bulgaria, August 20-24, 2006. Revised Papers. Lecture Notes in Computer Science 4310 Springer 2007, ISBN 978-3-540-70940-4 http://www.sigmod.org/publications/ $\mathrm{dblp} / \mathrm{db} / \mathrm{conf} / \mathrm{nma} / \mathrm{nma2006}$.html

[18] MMSC-2009 Special issue of Serdica Journal of Computing 4, 1, 2010) http://serdica-comp.math.bas.bg/index.php/ serdicajcomputing/issue/view/26 
[19] Markov, S. and V. Kreinovich, Special Session Set-Valued Numerics and Reliable Computing - Sozopol, Bulgaria, June 48, 2003, Reliable Computing 10, Number 1 / February, 2004, 59-61.

[20] Markov, S., On the Use of Computer Algebra Systems and Enclosure Methods in the Modelling and Optimization of Biotechnological Processes, Bioautomation (Intern. Electronic Journal) 3, 2005, 1-9.

[21] Markov, S., Biomathematics and interval analysis: a prosperous marriage, In: M. D. Todorov, Ch. I. Christov, Eds., AIP Conference Proceedings, Vol. 1301, Proc. 2nd International Conference on Applications of Mathematics in Technical and Natural Sciences, Sozopol, Bulgaria, June 21-26, 2010 (Amitans'2010), American Institute of Physics, Melville, New York, 2010, 26-36.

http://2010.eac4amitans.com/

[22] Milner, Fabio A., The International Congress on Deterministic and Stochastic Aspect of Biointeraction, Mathematical Biology Newsletter, Volume 11 \# 1, 1998.

http://www.smb.org/newsletter/11.1/index.shtml

[23] Cohen, J. E., Mathematics is Biology's next Microscope, only better; Biology is Mathematics' next Physics, only Better. PLoS Biol 2(12) (2004): e439.

doi:10.1371/journal.pbio.0020439

[24] Corpas, M., Gehlenborg N., Janga, S.C., Bourne, P.E. (2008), Ten Simple Rules for Organizing a Scientific Meeting. PLoS Comput. Biol. 4(6): e1000080. doi:10.1371/journal.pcbi.1000080

[25] Corpas, M., Scientists and societies. Nature. 2005;436:1204. doi:10.1038/nj70541204b.

[26] Biomath Forum Society. http://www.biomath.bg/

[27] Ivanov, T., Original Research Articles in Peer-Reviewed Journals from Past BIOMATH Conferences, Biomath Communications 1/1 http://www.biomathforum.org/biomath/ index.php/conference/article/view/348 
[28] Biomath 2011 Conference.

http://www.biomath.bg/2011/index.php

[29] Anguelov, R., S. Markov (Eds), Biomath-2011 Special Issue, Computers \& Mathematics with Applications 64, 3, 161-390 (August 2012)

[30] Biomath 2012 Conference. http://www. biomath.bg/2012/index.php

[31] Anguelov, R., S. Markov (Eds), Biomath-2012 Special Issue, Computers \& Mathematics with Applications 66, 9, 1533-1726 (November 2013)

[32] Beschkov, V., S. Markov (Eds), Biomath-2011 Special Issue, Biotechnology \& Biotechnological Equipment vol 2 (2) (2012), 3242-3320, BIOINFORMATICS (selected papers from BioMath 2011)

[33] Biomath 2013 Conference.

http://www. biomath.bg/2013/index.php

[34] Anguelov, R., S. Markov (Eds), Biomath-2012 Special Issue, Computers \& Mathematics with Applications 66, 9, 1533-1726 (November 2013)

[35] Biomath 2014 Conference.

http://www.biomath.bg/2014/index.php

[36] Biomath 2015 Conference.

http://www.biomath.bg/2015/index.php

[37] Biomath Forum Journal "BIOMATH".

http://www . biomathforum.org/biomath/index.php/biomath

[38] Biomath Forum Journal "Biomath Communications". http://www.biomathforum.org/biomath/index.php/conference

[39] Marsteller, P. et al., Toward Integration: From Quantitative Biology to Mathbio-Biomath?, CBE Life Sci Educ 9 (3), 165-171 (2010). doi: $10.1187 /$ cbe.10-03-0053 
[40] Jungck, J. R., Mathematical Biology Education: Modeling Makes Meaning, Mathematical Modelling of Natural Phenomena 6 (06), 1-21 (2011).

DOI: http://dx.doi.org/10.1051/mmnp/20116601

[41] Bergevin, Ch., Towards Improving the Integration of Undergraduate Biology and Mathematics Education, J. of Microbiology \& Biology Education 11, 2010, 28-33

DOI: $10.1128 /$ jmbe.v11i1.134

[42] Biomath in the schools, Book Series: DIMACS-Series in Discrete Mathematics and Theoretical Computer Science, Vol: 76 Pages: 109112, Published: 2011

[43] Robeva, R., ey al., An Invitation to Biomathematics, AP, 2008.

[44] Wikipedia. Mathematical and theoretical biology. http://en.wikipedia.org/wiki/ Mathematical_and_theoretical_biology 